\title{
THE
}

4-15-1996

\section{Order-Parameter Fluctuations in the Frustrated Heisenberg Model on the Square Lattice}

\author{
Shu Zhang \\ University of Rhode Island \\ Gerhard Müller \\ University of Rhode Island, gmuller@uri.edu
}

Follow this and additional works at: https://digitalcommons.uri.edu/phys_facpubs

Terms of Use

All rights reserved under copyright.

\section{Citation/Publisher Attribution}

Shu Zhang and Gerhard Müller. Order-parameter fluctuations in the frustrated Heisenberg model on a square lattice. J. Appl. Phys. 79 (1996), 6630-6632.

Available at: http://dx.doi.org/10.1063/1.361906

This Article is brought to you for free and open access by the Physics at DigitalCommons@URI. It has been accepted for inclusion in Physics Faculty Publications by an authorized administrator of DigitalCommons@URI. For more information, please contact digitalcommons-group@uri.edu. 


\title{
Order-parameter fluctuations in the frustrated Heisenberg model on the square lattice
}

\author{
Shu Zhang and Gerhard Müller \\ Department of Physics, The University of Rhode Island, Kingston, Rhode Island 02881-0817
}

\begin{abstract}
The $T=0$ dynamics of the two-dimensional $s=1 / 2$ Heisenberg model with competing nearest-neighbor $\left(J_{1}\right)$ and next-nearest-neighbor $\left(J_{2}\right)$ interactions is explored via the recursion method, specifically the frequency-dependent fluctuations of the order parameters associated with some of the known or suspected ordering tendencies in this system, i.e., Néel, collinear, dimer, and chiral order. The results for the dynamic structure factors of the respective fluctuation operators show a strong indication of collinear order at $J_{2} / J_{1} \gtrsim 0.6$ and a potential for dimer order at $0.5 \leqq J_{2} / J_{1} \lesssim 0.6$, whereas the chiral ordering tendency is observed to be considerably weaker.

(C) 1996 American Institute of Physics. [S0021-8979(96)00808-5]
\end{abstract}

The impact of quantum fluctuations on the zerotemperature phase diagram of a quantum many-body system tends to be strongest if that system contains competing interactions. A particular, sometimes exotic phase may be stabilized by quantum fluctuations in the presence of interactions that frustrate each other's ordering tendencies. The twodimensional (2D) Heisenberg antiferromagnet with nearestneighbor $(n n)$ and next-nearest-neighbor ( $n n n)$ coupling on the square lattice,

$H=J_{1} \sum_{\mathbf{r}} \mathbf{S}_{\mathbf{r}} \cdot\left(\mathbf{S}_{\mathbf{r}+\hat{\mathbf{x}}}+\mathbf{S}_{\mathbf{r}+\hat{\mathbf{y}}}\right)+J_{2} \sum_{\mathbf{r}} \mathbf{S}_{\mathbf{r}} \cdot\left(\mathbf{S}_{\mathbf{r}+\hat{\mathbf{x}}+\hat{\mathbf{y}}}+\mathbf{S}_{\mathbf{r}+\hat{\mathbf{x}}-\hat{\mathbf{y}}}\right)$,

has been a prominent object of study in this context. ${ }^{1}$

The Néel long-range order (LRO) present in the ground state of the $n n$ model disappears at some critical coupling ratio, $J_{2} / J_{1} \simeq 0.4$, and is replaced, at sufficiently large $J_{2} / J_{1}$, by Néel LRO within each of the two $n n n$ sublattices. The latter is preceeded, at $J_{2} / J_{1} \gtrsim 0.65$, by collinear LRO, which breaks the (discrete) rotational symmetry of $H$ on the lattice but not yet its (continuous) rotational symmetry in spin space. The Néel and collinear order parameters (OPs) are described by the operators

$$
O_{N}=\frac{1}{N} \sum_{\mathbf{r}}(-1)^{x+y} N_{\mathbf{r}}, \quad O_{C}=\frac{1}{N} \sum_{\mathbf{r}} C_{\mathbf{r}},
$$

where $N_{\mathbf{r}}=S_{\mathbf{r}}^{z}, C_{\mathbf{r}}=\mathbf{S}_{\mathbf{r}} \cdot\left(\mathbf{S}_{\mathbf{r}+\hat{\mathbf{x}}}+\mathbf{S}_{\mathbf{r}-\hat{\mathbf{x}}}-\mathbf{S}_{\mathbf{r}+\hat{\mathbf{y}}}-\mathbf{S}_{\mathbf{r}-\hat{\mathbf{y}}}\right)$.

At intermediate coupling ratios, $0.4 \leqq J_{2} / J_{1} \leqq 0.65$, the Néel and collinear ordering tendencies keep each other at bay and thus make the frustrated ground state susceptible to different kinds of ordering potentialities. Dimer order, 2,3 twist order, ${ }^{4}$ and chiral order ${ }^{5}$ have been proposed in this context. The dimer and chiral OPs considered here are defined by the following expressions in terms of spin operators:

$$
O_{D}=\frac{1}{N} \sum_{\mathbf{r}}(-1)^{x} D_{\mathbf{r}}, \quad O_{\chi}=\frac{1}{N} \sum_{\mathbf{r}} \chi_{\mathbf{r}},
$$

where $D_{\mathbf{r}}=\mathbf{S}_{\mathbf{r}} \cdot \mathbf{S}_{\mathbf{r}+\hat{\mathbf{x}}}, \quad \chi_{\mathbf{r}}=Z_{\mathbf{r}, \mathbf{r}+\hat{\mathbf{x}}, \mathbf{r}+\hat{\mathbf{x}}+\hat{\mathbf{y}}}-Z_{\mathbf{r}, \mathbf{r}+\hat{\mathbf{x}}+\hat{\mathbf{y}}, \mathbf{r}+\hat{\mathbf{y}}}$, $Z_{\mathbf{i}, \mathbf{j}, \mathbf{k}}=i\left(S_{\mathbf{i}}^{+} S_{\mathbf{j}}^{-}-S_{\mathbf{i}}^{-} S_{\mathbf{j}}^{+}+S_{\mathbf{j}}^{+} S_{\mathbf{k}}^{-}-S_{\mathbf{j}}^{-} S_{\mathbf{k}}^{+}+S_{\mathbf{k}}^{+} S_{\mathbf{i}}^{-}-S_{\mathbf{k}}^{-} S_{\mathbf{i}}^{+}\right)$. They probe the long-range phase coherence of singlets stacked in columns along the $y$ axis and the handedness of the spin configuration on a plaquette, respectively, in the ground-state wave function $|G\rangle$. Whether any one of these types of LRO is, in fact, realized, or whether a ground state with short-range correlations of the resonating-valence-bond type, ${ }^{6,7}$ for example, is stabilized, has not been determined for certain.

The absence or presence of a specific type of LRO determines whether the associated OP correlation function decays to zero or not. In the finite-cluster data, the relevant information on the asymptotic behavior is best captured by the expectation value of the squared OP. The problem here is to find a meaningful reference point for any enhancement in that quantity. ${ }^{3}$

An alternative avenue to comparing the different ordering tendencies in the spin-frustrated ground state of (1) is to explore the dynamic (i.e., frequency-dependent) fluctuations of any proposed OP. The recursion $\operatorname{method}^{8}$ in conjunction with recently developed techniques of continued-fraction analysis $^{9,10}$ is very suitable for that purpose. Here the dynamical information is derived from the finite-size groundstate wave function. No excited states have to be computed. This is an important advantage for the study of systems with complicated spectra and with potential OPs that have widely varying symmetry properties, as is the case here.

We investigate the fluctuations of the four OPs defined in (2) and (3) as they manifest themselves in the dynamic structure factors

$$
S_{A A}(\mathbf{q}, \omega)=\int_{-\infty}^{+\infty} d t e^{i \omega t}\left\langle F_{\mathbf{q}}^{A}(t) F_{-\mathbf{q}}^{A}\right\rangle
$$

where $F_{\mathbf{q}}^{A}=N^{-1 / 2} \Sigma_{\mathbf{r}} e^{i \mathbf{q} \cdot \mathbf{r}} A_{\mathbf{r}}$ is the fluctuation operator associated with a given $\mathrm{OP}$, and $A_{\mathbf{r}}$ stands for $N_{\mathbf{r}}, C_{\mathbf{r}}, D_{\mathbf{r}}$, or $\chi_{\mathbf{r}}$, as defined above.

The recursion algorithm in the present context is based on an orthogonal expansion of the wave function $\left|\Psi_{\mathbf{q}}^{A}(t)\right\rangle$ $=F_{\mathbf{q}}^{A}(-t)|G\rangle$. It produces (after some intermediate steps) a sequence of continued-fraction coefficients $\Delta_{1}^{A}(\mathbf{q})$, $\Delta_{2}^{A}(\mathbf{q}), \ldots$ for the relaxation function

$$
c_{0}^{A A}(\mathbf{q}, z)=\frac{1}{z+\frac{\Delta_{1}^{A}(\mathbf{q})}{z+\frac{\Delta_{2}^{A}(\mathbf{q})}{z+\ldots}},}
$$




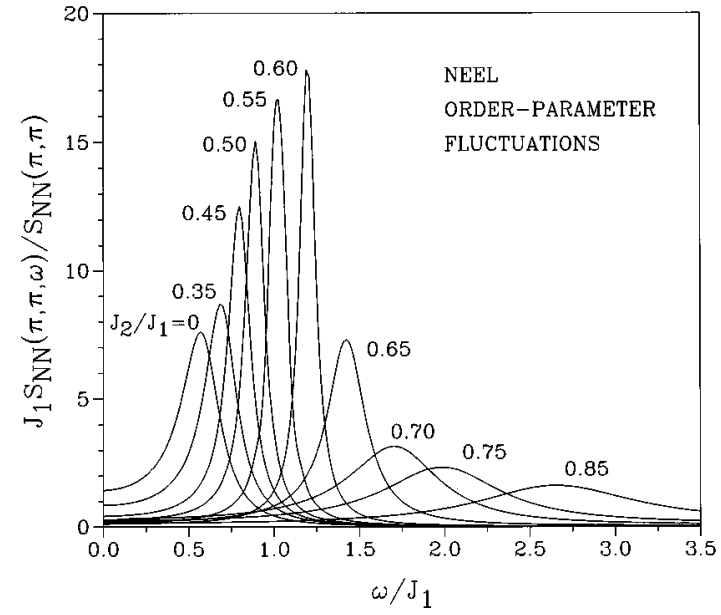

FIG. 1. $T=0$ dynamic structure factor (4) normalized by its integrated intensity for the Néel fluctuation operator $F_{\mathbf{q}}^{N}$ at $\mathbf{q}=(\pi, \pi)$ of the Hamiltonian (1) at various values of the coupling ratio $J_{2} / J_{1}$, obtained via strongcoupling continued-fraction reconstruction from the coefficients $\Delta_{1}, \ldots, \Delta_{6}$ and a Gaussian terminator as explained in Refs. 9 and 10.

which is the Laplace transform of the symmetrized correlation function $\mathfrak{R}\left\langle F_{\mathbf{q}}^{A}(t) F_{-\mathbf{q}}^{A}\right\rangle /\left\langle F_{\mathbf{q}}^{A} F_{-\mathbf{q}}^{A}\right\rangle$. The $T=0$ dynamic structure factor is then obtained from (5) via

$$
S_{A A}(\mathbf{q}, \omega)=4\left\langle F_{\mathbf{q}}^{A} F_{-\mathbf{q}}^{A}\right\rangle \Theta(\omega) \lim _{\varepsilon \rightarrow 0} \Re\left[c_{0}^{A A}(\mathbf{q}, \varepsilon-i \omega)\right],
$$

where $\left\langle F_{\mathbf{q}}^{A} F_{-\mathbf{q}}^{A}\right\rangle=S_{A A}(\mathbf{q})$ is the integrated intensity. ${ }^{11}$

All the results presented here are for clusters of $N=4 \times 4$ sites with periodic boundary conditions. Extreme care must be exercised in separating finite-size effects from properties that reflect the physics of the infinite system. This distinction can be made with more confidence for coupling ratios $J_{2} / J_{1} \lesssim 0.7$ than at $J_{2} / J_{1}>0.7$, where the gradual decoupling of the two $n n n$ sublattices causes a crossover in the finite-size effects.

The Néel OP fluctuations are probed by the dynamic structure factor $S_{N N}(\mathbf{q}, \omega)$ at the wave vector $\mathbf{q}=(\pi, \pi)$. This quantity is shown in Fig. 1 for various coupling ratios. The presence of Néel LRO at $J_{2} / J_{1}=0$ implies that $S_{N N}(\pi, \pi, \omega)$ is governed by a zero-frequency peak. Quantum fluctuations split the ground-state level for finite $N$. In a $4 \times 4$ cluster the finite-size gap is known to be of magnitude $\Delta E / J_{1} \simeq 0.57{ }^{10}$ The peak position of the curve for $J_{2} / J_{1}=0$ must be interpreted with this fact in mind.

What are the effects of the $n n n$ coupling on the Néel OP fluctuations? For $0 \leqslant J_{2} / J_{1} \leq 0.4$, i.e., over the estimated range of the Néel phase, we observe only small changes in peak position and line shape. Then the Néel OP fluctuations begin to change rapidly in two stages:

(i) Over the range $0.4 \leq J_{2} / J_{1} \leqslant 0.6$, the peak position moves to higher frequencies at an accelerated rate, the linewidth shrinks, and the integrated intensity (not shown) drops to $32 \%$ of its value at $J_{2} / J_{1}=0$. This signals the presence of some non-Néel type ordering tendency which supports welldefined Néel modes at increasingly high frequencies.

(ii) At $J_{2} / J_{1} \geq 0.6$ the linewidth of $S_{N N}(\pi, \pi, \omega)$ grows rapidly, while the peak position moves further up and the

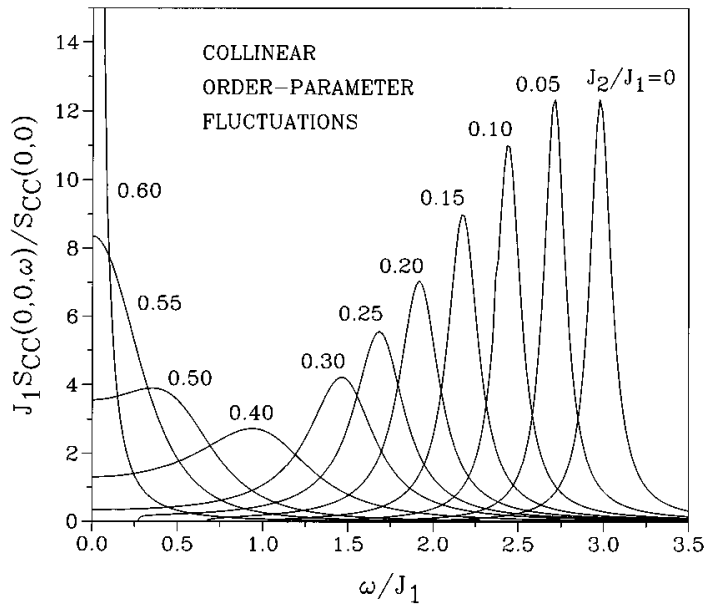

FIG. 2. $T=0$ dynamic structure factor (4) normalized by its integrated intensity for the collinear fluctuation operator $F_{\mathbf{q}}^{C}$ at $\mathbf{q}=(0,0)$ of the Hamiltonian (1) at various values of the coupling ratio $J_{2} / J_{1}$, obtained via strongcoupling continued-fraction reconstruction from the coefficients $\Delta_{1}, \ldots, \Delta_{6}$ and a Gaussian terminator.

integrated intensity continues to fade away quickly. As the two $n n n$ sublattices begin to decouple, the system ceases to support well-defined Néel modes.

The dynamic structure factor $S_{C C}(0,0, \omega)$, which describes the collinear OP fluctuations, is shown in Fig. 2. At $J_{2} / J_{1}=0$, we observe a fairly sharp collinear mode at $\omega / J_{1} \simeq 3.0$. As the Néel ordering tendency weakens with increasing $J_{2} / J_{1}$, the collinear mode shifts to lower frequencies, while its line shape broadens considerably. The width reaches a maximum at $J_{2} / J_{1} \simeq 0.4$. Between here and $J_{2} / J_{1} \simeq 0.55$, where the competing dimer and chiral ordering tendencies are at their peak, the collinear mode moves to $\omega=0$, and the integrated intensity more than triples in relation to its value at $J_{2} / J_{1}=0$. In the interval $0.55 \lesssim J_{2} / J_{1}$ $\lesssim 0.7$, the function $S_{C C}(0,0, \omega)$ transforms into a narrow

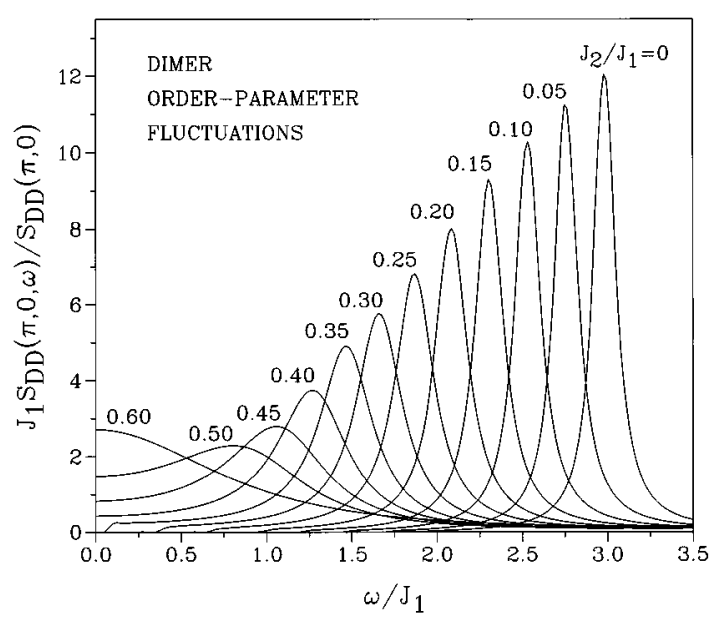

FIG. 3. $T=0$ dynamic structure factor (4) normalized by its integrated intensity for the dimer fluctuation operator $F_{\mathbf{q}}^{D}$ at $\mathbf{q}=(\pi, 0)$ of the Hamiltonian (1) at various values of the coupling ratio $J_{2} / J_{1}$, obtained via strongcoupling continued-fraction reconstruction from the coefficients $\Delta_{1}, \ldots, \Delta_{6}$ and a Gaussian terminator. 


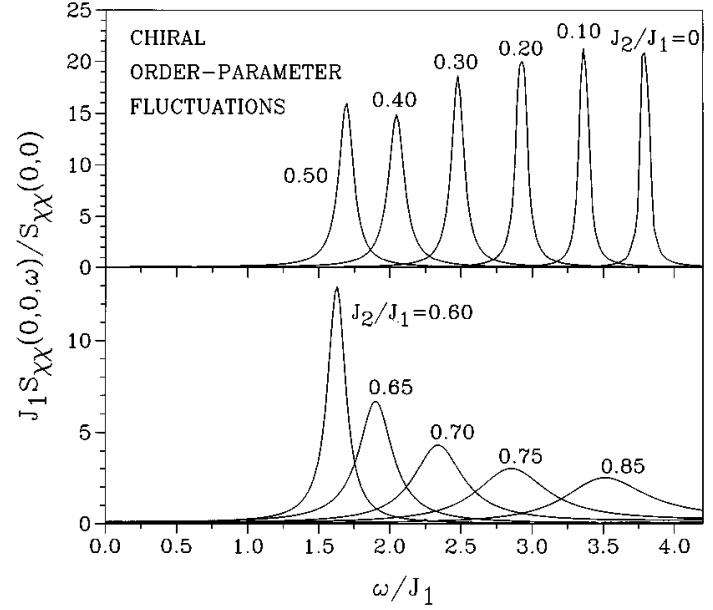

FIG. 4. $T=0$ dynamic structure factor (4) normalized by its integrated intensity for the chiral fluctuation operator $A_{\mathbf{q}}^{\chi}$ at $\mathbf{q}=(0,0)$ of the Hamiltonian (1) at various values of the coupling ratio $J_{2} / J_{1}$, obtained via strongcoupling continued-fraction reconstruction from the coefficients $\Delta_{1}, \ldots, \Delta_{6}$ and a Gaussian terminator.

central peak, and $S_{C C}(0,0)$ increases by another factor of $\simeq 1.6$. This clearly reflects the onset of collinear LRO in the infinite system.

The dimer OP fluctuations as described by the dynamic structure factor $S_{D D}(\pi, 0, \omega)$ and shown in Fig. 3 resemble those of the collinear OP with respect to line shape and peak position for as long as the Néel ordering tendency is perceptible in the ground state $\left(J_{2} / J_{1} \leqq 0.4\right)$. Both modes become soft and very broad at $J_{2} / J_{1} \simeq 0.55$, but then they part company. While the collinear mode has been observed to transform into a high-intensity narrow central peak, the dimer mode, which has reached its maximum intensity here $(\simeq 1.8$ times its value at $J_{2} / J_{1}=0$ ), broadens further and loses intensity very rapidly. It literally dissolves as the intersublattice correlations begin to weaken at $J_{2} / J_{1} \geq 0.7$. Nevertheless, the softness of the dimer OP fluctuations at $J_{2} / J_{1} \simeq 0.55$ in the $4 \times 4$ cluster is consistent with dimer LRO in the infinite system.
The chiral OP fluctuations are based more significantly on intra-sublattice correlations than the dimer OP fluctuations and, therefore, evolve differently. This is illustrated in Fig. 4. At $J_{2} / J_{1}=0$ the dynamic structure factor $S_{\chi \chi}(0,0, \omega)$ exhibits a sharp mode at $\omega / J_{1} \simeq 3.8$. At $J_{2} / J_{1}$ $\simeq 0.55$, the peak position has moved down to $\omega / J_{1} \simeq 1.6$, while the linewidth has increased only slightly, and the integrated intensity has grown to a maximum value of $\simeq 2.3$ times its value at $J_{2} / J_{1}=0$. Then the peak position starts to move back out to higher frequencies, the line shape begins to broaden, but less so compared to that of the dimer fluctuations, and the integrated intensity drops rapidly. The minimum gap of the chiral mode is perhaps too large to be entirely attributable to a finite-size effect, which would indicate that the observed chiral ordering tendency does not turn into chiral LRO as $N \rightarrow \infty$.

This work was supported by NSF Grant No. DMR-9312252 and by the NCSA at Urbana-Champaign.

${ }^{1}$ J. Richter, Phys. Rev. B 47, 5794 (1993). This paper contains many references to pertinent studies.

${ }^{2}$ M. P. Gelfand, R. R. P. Singh, and D. A. Huse, Phys. Rev. B 40, 10801 (1989); A. V. Chubukov and Th. Jolicoeur, ibid. 44, 12050 (1991).

${ }^{3}$ R. R. P. Singh and R. Narayanan, Phys. Rev. Lett. 65, 1072 (1990).

${ }^{4}$ E. Dagotto and A. Moreo, Phys. Rev. Lett. 63, 2148 (1989).

${ }^{5}$ J. Richter, C. Gros, and W. Weber, Phys. Rev. B 44, 906 (1991); 45, 10113 (1992).

${ }^{6}$ P. Chandra and B. Doucot, Phys. Rev. B 38, 9335 (1988).

${ }^{7}$ J. Richter, Z. Phys. B 79, 403 (1990).

${ }^{8}$ R. Haydock, Solid State Phys. 35, 215 (1980); M. H. Lee, Phys. Rev. B 26, 2547 (1982); E. R. Gagliano and C. A. Balseiro, ibid. 38, 11766 (1988).

${ }^{9}$ V. S. Viswanath and G. Müller, Recursion Method - Application to Many-Body Dynamics, Lecture Notes in Physics, Vol. 23 (Springer, New York, 1994).

${ }^{10}$ V. S . Viswanath, S. Zhang, J. Stolze, and G. Müller, Phys. Rev. B 49, 9702 (1994)

${ }^{11}$ A graph of the integrated intensity vs $J_{2} / J_{1}$ for each of the four types of OP fluctuations depicted in Figs. 1 through 4 can be found, e.g., in Ref. 1 (with different $N$-dependent factors). 